\title{
A LOCAL-GLOBAL PRINCIPLE FOR LINEAR DEPENDENCE OF NONCOMMUTATIVE POLYNOMIALS
}

\author{
MATEJ BREŠAR ${ }^{1}$ AND IGOR KLEP ${ }^{2}$
}

\begin{abstract}
A set of polynomials in noncommuting variables is called locally linearly dependent if their evaluations at tuples of matrices are always linearly dependent. By a theorem of Camino, Helton, Skelton and Ye, a finite locally linearly dependent set of polynomials is linearly dependent. In this short note an alternative proof based on the theory of polynomial identities is given. The method of the proof yields generalizations to directional local linear dependence and evaluations in general algebras over fields of arbitrary characteristic. A main feature of the proof is that it makes it possible to deduce bounds on the size of the matrices where the (directional) local linear dependence needs to be tested in order to establish linear dependence.
\end{abstract}

\section{INTRODUCTION}

As part of the studies in free analysis motivated from systems engineering, Camino, Helton, Skelton and Ye [CHSY] consider local linear dependence of functions in noncommuting (nc) variables, e.g. polynomials and rational functions. One of the core results of [CHSY] is that locally linearly dependent nc polynomials are (globally) linearly dependent. This result is what we call the local-global principle for linear dependence (of polynomials). It has been exploited repeatedly since; often in connection with noncommutative convexity and geometry, cf. [HHLM, DHM, GHV]. We also refer to the tutorial $[\mathrm{HKM}]$ for a more streamlined presentation of the proof and its applications.

Our aim is to give an algebraists' response to [CHSY]. That is, we give a proof of this local-global principle that is motivated by the theory of polynomial identities. As such it applies not only to matrix algebras but to evaluations in general algebras over fields of arbitrary characteristic. However, even in the case of matrix algebras it allows us to extract additional information, e.g. the size of the matrices where the local linear dependence needs to be checked in order to establish linear dependence. Also, we establish bounds in the case of directional dependence (see below for definitions and precise statements), something the original proofs do not allow.

This note is organized as follows. After a preliminary Section 2 introducing all the notions needed, we give our main results in Section 3. As this is an algebraic paper addressed also to analysts, we will give a somewhat detailed treatment of the algebraic tools that will be used in our proofs.

Date: 8 March 2011.

2010 Mathematics Subject Classification. Primary 16R50, 08B20, Secondary 16W10.

Key words and phrases. Noncommutative polynomial, free algebra, linear dependence, local linear dependence, polynomial identity, involution.

${ }^{1}$ Supported by the Slovenian Research Agency (Program No. P1-0288). ${ }^{2}$ Supported by the Slovenian Research Agency (Project No. J1-3608 and Program No. P1-0222). 


\section{PRELIMINARIES}

2.1. Notation and set-up. In this section we fix the basic notation and terminology we shall use throughout the paper. Let $\mathbb{F}$ be a field.

2.1.1. Free algebra. By $\mathbb{F}\langle\underline{X}\rangle$ we denote the free associative algebra generated by $\underline{X}=\left\{X_{1}, X_{2}, \ldots\right\}$, i.e., the algebra of all polynomials in noncommuting variables $X_{i}$. We write $\langle\underline{X}\rangle$ for the monoid freely generated by $\underline{X}$, i.e., $\langle\underline{X}\rangle$ consists of words in the letters $X_{1}, X_{2}, \ldots$ (including the empty word denoted by 1 ). Write $\mathbb{F}\langle\underline{X}\rangle_{k}$ for the vector space consisting of the polynomials of degree at most $k$. Sometimes, for notational convenience, we shall also use $Y_{j}, Z_{j}$ to denote noncommuting variables.

2.1.2. Evaluations and representations. If $p \in \mathbb{F}\left\langle X_{1}, \ldots, X_{n}\right\rangle, \mathcal{A}$ is an $\mathbb{F}$-algebra, and $\underline{a} \in \mathcal{A}^{n}$, then $p(\underline{a}) \in \mathcal{A}$ is the evaluation of $p$ at $\underline{a}$. This gives rise to a representation $\mathrm{ev}_{\underline{a}}: \mathbb{F}\left\langle X_{1}, \ldots, X_{n}\right\rangle \rightarrow \mathcal{A}$.

2.1.3. Directional evaluations. Suppose $\mathcal{A}$ is a subalgebra of an endomorphism algebra $\operatorname{End}(V)$ for an $\mathbb{F}$-vector space $V$. Given a polynomial $p \in \mathbb{F}\left\langle X_{1}, \ldots, X_{n}\right\rangle$, an $n$-tuple $\underline{a} \in \mathcal{A}^{n}$ and $v \in V$, the expression $p(\underline{a}) v$ is called the directional evaluation of $p$ in the direction $(\underline{a}, v)$.

2.2. Polynomial identities. We say that $p \in \mathbb{F}\left\langle X_{1}, \ldots, X_{n}\right\rangle$ is an identity of an $\mathbb{F}$-algebra $\mathcal{A}$ if $p(\underline{a})=0$ for all $\underline{a} \in \mathcal{A}^{n}$. If $p \neq 0$, then $p$ is called a polynomial identity of $\mathcal{A}$. For example, $\mathcal{A}$ is commutative if and only if $\mathrm{St}_{2}:=X_{1} X_{2}-X_{2} X_{1}$ is its polynomial identity. We say that $\mathcal{A}$ is a PI-algebra if there exists a polynomial identity of $\mathcal{A}$. Obviously, subalgebras and homomorphic images of PI-algebras are again PI-algebras. Besides commutative algebras, the simplest examples of PIalgebras are finite dimensional ones. To see this, we introduce, for every $n \in \mathbb{N}$, the so-called standard polynomial $\mathrm{St}_{n}=\operatorname{St}_{n}\left(X_{1}, \ldots, X_{n}\right)$ by

$$
\mathrm{St}_{n}:=\sum_{\pi \in S_{n}} \operatorname{sign}(\pi) X_{\pi(1)} \ldots X_{\pi(n)}
$$

where $S_{n}$ is the symmetric group of degree $n$. It is easy to see that

$$
\operatorname{St}_{n}\left(X_{1}, \ldots, X_{i}, \ldots, X_{i}, \ldots, X_{n}\right)=0,
$$

i.e., $\mathrm{St}_{n}$ vanishes if two variables are the same. Accordingly, $\operatorname{St}_{n}\left(a_{1}, \ldots, a_{n}\right)=0$ whenever $a_{1}, \ldots, a_{n}$ are linearly dependent elements from an algebra $\mathcal{A}$. This in particular shows that $\mathrm{St}_{n}$ is a polynomial identity of every algebra $\mathcal{A}$ with $\operatorname{dim}_{\mathbb{F}} \mathcal{A}<$ $n$. Thus, $\mathrm{St}_{d^{2}+1}$ is a polynomial identity of the matrix algebra $M_{d}(\mathbb{F})$. There is, however, a much better result, the Amitsur-Levitzki theorem, saying that $\mathrm{St}_{2 d}$ is a polynomial identity of $M_{d}(\mathbb{F})$; moreover, it is a polynomial identity of $M_{d}(\mathcal{C})$ where $\mathcal{C}$ is an arbitrary commutative algebra. The number $2 d$ cannot be lowered: a bit tricky, but elementary argument shows that a polynomial of degree $<2 d$ is never a polynomial identity of $M_{d}(\mathbb{F})$. Therefore a polynomial that was a polynomial identity of $M_{d}(\mathbb{F})$ for every $d$ does not exist. This implies that the algebra of all linear operators on an infinite dimensional vector space (which contains isomorphic copies of all $M_{d}(\mathbb{F})$ as its subalgebras) is not a PI-algebra. In fact, under mild assumptions a PI-algebra is quite close to a matrix algebra. For instance, it turns out that every prime PI-algebra can be embedded into $M_{d}(\mathbb{K})$ for some $d \geq 1$, where $\mathbb{K}$ is a field extension of the base field $\mathbb{F}$. Recall that an algebra $\mathcal{A}$ is said to be prime if the product of any of its two nonzero ideals is nonzero.

Let us also mention a notion related to a polynomial identity: we say that $p \in \mathbb{F}\left\langle X_{1}, \ldots, X_{n}\right\rangle$ is a central polynomial on $M_{d}(\mathbb{F})$ if $p \neq 0, p$ is not a polynomial 
identity, and $p(\underline{A})$ is a scalar multiple of the identity matrix for every $\underline{A} \in M_{d}(\mathbb{F})^{n}$. For instance, $\left(X_{1} X_{2}-X_{2} X_{1}\right)^{2}$ is a central polynomial of $M_{2}(\mathbb{F})$, as one can easily check. It is much harder to find central polynomials on $M_{d}(\mathbb{F})$ for larger $d$ 's. Anyhow, it is a fact that they do exist for every $d$.

For full accounts on polynomial identities we refer the reader to [Pro] and [Row].

2.3. Capelli polynomials and a theorem of Razmyslov. For $n \in \mathbb{N}$ we define the Capelli polynomial $C_{2 n-1}=C_{2 n-1}\left(X_{1}, \ldots, X_{2 n-1}\right)$ as follows:

$$
C_{2 n-1}:=\sum_{\pi \in S_{n}} \operatorname{sign}(\pi) X_{\pi(1)} X_{n+1} X_{\pi(2)} X_{n+2} \cdots X_{\pi(n-1)} X_{2 n-1} X_{\pi(n)} .
$$

For example, $C_{3}=X_{1} X_{3} X_{2}-X_{2} X_{3} X_{1}$. Note that by formally replacing all $X_{j}$, $j>n$, by $1, C_{2 n-1}$ reduces to $\mathrm{St}_{n}$. Just as for the standard polynomials, one can check that

$$
C_{2 n-1}\left(X_{1}, \ldots, X_{i}, \ldots, X_{i}, \ldots, X_{n}, X_{n+1}, \ldots, X_{2 n-1}\right)=0,
$$

implying that for elements $a_{i}, b_{i}$ from an algebra $\mathcal{A}$ we have

$$
C_{2 n-1}\left(a_{1}, \ldots, a_{n}, b_{1}, \ldots, b_{n-1}\right)=0
$$

whenever $a_{1}, \ldots, a_{n}$ are linearly dependent. An important theorem of Razmyslov (cf. [BMM, Theorem 2.3.7] or [Row, Theorem 7.6.16]) states that the converse of this observation holds in a rather large class of algebras:

Theorem 2.1. Let $\mathcal{A}$ be a centrally closed prime algebra. Then $a_{1}, \ldots, a_{n} \in \mathcal{A}$ are linearly dependent if and only if $C_{2 n-1}\left(a_{1}, \ldots, a_{n}, b_{1}, \ldots, b_{n-1}\right)=0$ for all $b_{j} \in \mathcal{A}$.

The definition of a centrally closed prime algebra is too technical to be included here. The reader is referred to $[\mathrm{BMM}]$ for a detailed, or to $[\mathrm{BCM}]$ for an informal survey on this notion. Let us just mention what is relevant for our applications of Theorem 2.1: the free algebra $\mathbb{F}\langle\underline{X}\rangle$ is a centrally closed prime algebra (cf. [BMM, Theorem 2.4.4]).

Let us conclude this section by mentioning that we have used Razmyslov's Theorem 2.1 before - in [BK, Section 5.5] to prove a tracial multilinear Nullstellensatz.

2.4. Locally linearly dependent operators. Let $U$ and $V$ be vector spaces over $\mathbb{F}$. We say that linear operators $T_{1}, \ldots, T_{m}: U \rightarrow V$ are locally linearly dependent if $T_{1} u, \ldots, T_{m} u$ are linearly dependent vectors in $V$ for every $u \in U$. This does not necessarily mean that $T_{1}, \ldots, T_{m}$ are linearly dependent operators. Say, if $T_{1}$ and $T_{2}$ are rank one operators with the same range, then they are obviously locally linearly dependent, but not necessarily linearly dependent. Another example: if $\operatorname{dim}_{F} V<m$, then any linear operators $T_{1}, \ldots, T_{m}: U \rightarrow V$ are locally linearly dependent, but there is no reason to believe that they are linearly dependent.

The following result shows that the local linear dependence is intimately connected with the finite rank condition.

Theorem 2.2. If $T_{1}, \ldots, T_{m}: U \rightarrow V$ are locally linearly dependent operators, then then there exist $\alpha_{1}, \ldots, \alpha_{m} \in \mathbb{F}$, not all zero, such that $S=\alpha_{1} T_{1}+\cdots+\alpha_{m} T_{m}$ satisfies $\operatorname{rank} S \leq m-1$. This inequality is sharp.

It seems that the first result of this kind was obtained by Amitsur [Ami], however, with a conclusion that $\operatorname{rank} S \leq\left(\begin{array}{c}m+1 \\ 2\end{array}\right)-1$. For $\mathbb{F}=\mathbb{C}$ Theorem 2.2 was proved by Aupetit [Aup], for $\mathbb{F}$ an infinite field by Brešar and Šemrl [BŠ], and finally for $\mathbb{F}$ a finite field by Meshulam and Šmrl [MŠ]. The form in which we shall apply Theorem 2.2 is as follows: 
Lemma 2.3. Keep the assumptions of Theorem 2.2 and assume $U=V$. Then the rank of $C_{2 m-1}\left(T_{1}, \ldots, T_{m}, D_{1}, \ldots, D_{m-1}\right)$ cannot exceed $(m-1) m$ ! for any linear $D_{j}: U \rightarrow U$.

Proof. By Theorem 2.2 we may without loss of generality assume $T_{m}$ is a linear combination of $T_{1}, \ldots, T_{m-1}$ plus an operator $E$ of rank $\leq m-1$, e.g.,

$$
T_{m}=E+\sum_{j=1}^{m-1} \alpha_{j} T_{j}
$$

for some scalars $\alpha_{j}$. Then

$$
\begin{aligned}
C_{2 m-1}\left(T_{1}, \ldots, T_{m-1}, T_{m}, D_{1}, \ldots, D_{m-1}\right) & \\
= & C_{2 m-1}\left(T_{1}, \ldots, T_{m-1}, E+\sum_{j=1}^{m-1} \alpha_{j} T_{j}, D_{1}, \ldots, D_{m-1}\right) .
\end{aligned}
$$

Since $C_{2 m-1}$ is linear in each variable, it follows that

$C_{2 m-1}\left(T_{1}, \ldots, T_{m-1}, T_{m}, D_{1}, \ldots, D_{m-1}\right)=C_{2 m-1}\left(T_{1}, \ldots, T_{m-1}, E, D_{1}, \ldots, D_{m-1}\right)$.

The right-hand side of (2.1) is equal to a sum of $m$ ! operators, each of which has rank $\leq m-1$. This yields the desired bound.

Remark 2.4. Let us add that if $A: U \rightarrow U$ is a linear operator of rank $r$, then $A, A, \ldots, A^{r+1}$ are linearly dependent. This is well-known, but let us give a short proof for the sake of completeness. Consider the induced mapping

$$
\check{A}: U / \operatorname{ker}(A) \rightarrow \operatorname{ran}(A) .
$$

Since $\operatorname{dim}(U / \operatorname{ker}(A))=\operatorname{dim}(\operatorname{ran}(A))=r$, the characteristic polynomial

$$
\operatorname{charPoly}_{\check{A}}=a_{0}+a_{1} \lambda+\cdots+(-1)^{r} \lambda^{r} \in \mathbb{F}[\lambda]
$$

is of degree $r$ and kills $\check{A}$ by the Cayley-Hamilton theorem. Thus

$$
a_{0} A+a_{1} A^{2}+\cdots+(-1)^{r} A^{r+1}=0 .
$$

2.5. Local (directional) linear dependence of polynomials. Let $\mathcal{A}$ be an $\mathbb{F}$ algebra and let $S \subseteq \mathbb{F}\langle\underline{X}\rangle$.

(1) We say that $S$ is $\mathcal{A}$-locally linearly dependent if the elements

$$
\{p(\underline{A}) \mid p \in S\} \subseteq \mathcal{A}
$$

are linearly dependent for every $\underline{A} \in \mathcal{A}^{\mathbb{N}}$.

(2) Now suppose $\mathcal{A}$ is a subalgebra of $\operatorname{End}(V)$ for an $\mathbb{F}$-vector space $V$. We say that $S$ is $\mathcal{A}$-locally directionally linearly dependent if the vectors

$$
\{p(\underline{A}) v \mid p \in S\} \subseteq V
$$

are linearly dependent for every $\underline{A} \in \mathcal{A}^{\mathbb{N}}$ and $v \in V$.

(3) $S$ is (globally) linearly dependent if it is linearly dependent in $\mathbb{F}\langle\underline{X}\rangle$, i.e., there are $\alpha_{s} \in \mathbb{F}(s \in S)$, of which finitely many are nonzero but not all are zero, such that

$$
0=\sum_{s \in S} \alpha_{s} s
$$


Our core example is $\mathcal{A}=M_{d}(\mathbb{F})$, but our methods allow us to consider evaluations in general algebras. For instance, in Section 3.1 we establish that a finite set $S$ of polynomials is $\mathcal{A}$-locally linearly dependent for a non-PI algebra $\mathcal{A}$ if and only if $S$ is linearly dependent. In fact, the same conclusion holds whenever $S$ is $M_{d}(\mathbb{F})$-locally (directionally) linearly dependent for some $d$ large enough. As a side product of our proofs we establish bounds on $d$.

Remark 2.5. The notion of local (directional) linear dependence in free algebras is nontrivial. For example, if $\mathcal{A}$ is an $n$-dimensional algebra and $S \subseteq \mathbb{F}\langle\underline{X}\rangle$ is any set with $|S|>n$, then $S$ is $\mathcal{A}$-locally linearly dependent. Another example: any two central polynomials for $d \times d$ matrices are $M_{d}(\mathbb{F})$-locally linearly dependent, although they need not be linearly dependent in the free algebra.

\section{RESUlts}

3.1. Local linear dependence. We begin with one of the two of our main results.

Theorem 3.1. Let $\mathcal{A}$ be an $\mathbb{F}$-algebra and let $f_{1}, \ldots, f_{m} \in \mathbb{F}\left\langle X_{1}, \ldots, X_{n}\right\rangle$ be $\mathcal{A}$-locally linearly dependent. If $\mathcal{A}$ does not satisfy a polynomial identity of degree

$$
\beta:=\sum_{j} \operatorname{deg}\left(f_{j}\right)+m-1,
$$

then $f_{1}, \ldots, f_{m}$ are linearly dependent.

Proof. For all $\underline{a} \in \mathcal{A}^{n}$, the elements $f_{1}(\underline{a}), \ldots, f_{m}(\underline{a})$ are linearly dependent. Hence

$$
C_{2 m-1}\left(f_{1}(\underline{a}), \ldots, f_{m}(\underline{a}), b_{1}, \ldots, b_{m-1}\right)=0
$$

for all $b_{j} \in \mathcal{A}$. That is, $C_{2 m-1}\left(f_{1}, \ldots, f_{m}, Y_{1}, \ldots, Y_{m-1}\right)$ is an identity of $\mathcal{A}$. Since the degree of this polynomial is $\sum_{j} \operatorname{deg}\left(f_{j}\right)+m-1$, it follows from our assumption that $C_{2 m-1}\left(f_{1}, \ldots, f_{m}, Y_{1}, \ldots, Y_{m-1}\right)=0$. As the $f_{i}$ 's do not depend on $Y_{1}, \ldots, Y_{m-1}$, this trivially yields an apparently stronger conclusion

$$
C_{2 m-1}\left(f_{1}, \ldots, f_{m}, h_{1}, \ldots, h_{m-1}\right)=0
$$

for all $h_{1}, \ldots, h_{m-1} \in \mathbb{F}\langle\underline{X}\rangle$. Hence by Theorem 2.1, applied to the algebra $\mathbb{F}\langle\underline{X}\rangle$, $f_{1}, \ldots, f_{m}$ are linearly dependent.

The bound $\beta$ may be sometimes too big, but in general it cannot be improved.

Example 3.2. Let $f_{1}$ be a polynomial identity of $\mathcal{A}$ of minimal degree. The set $\left\{f_{1}\right\}$ is then linearly independent and $\mathcal{A}$-locally linearly dependent. In this case $\beta=\operatorname{deg} f_{1}$, so that $\mathcal{A}$ satisfies a polynomial identity of degree $\beta$, and does not satisfy a polynomial identity of degree $<\beta$.

Example 3.3. Let $\mathcal{A}=\mathbb{F}$. Let $f_{1}=X_{1}$ and $f_{2}=1$. Obviously, the set $\left\{f_{1}, f_{2}\right\}$ is linearly independent and $\mathcal{A}$-locally linearly dependent, $\beta=2, \mathcal{A}$ satisfies a polynomial identity of degree 2 , and does not satisfy a polynomial identity of degree $<2$.

Corollary 3.4. Let $\mathcal{A}$ be a non-PI algebra and let $S \subseteq \mathbb{F}\langle\underline{X}\rangle$ be an $\mathcal{A}$-locally linearly dependent finite set of polynomials. Then $S$ is linearly dependent.

Since $M_{s}(\mathbb{F})$ does not satisfy a polynomial identity of degree $<2 s$, we also have the following corollary. 
Corollary 3.5. Let $f_{1}, \ldots, f_{m} \in \mathbb{F}\left\langle X_{1}, \ldots, X_{n}\right\rangle$ be $M_{s}(\mathbb{F})$-locally linearly dependent for some

$$
s>\frac{1}{2}\left(\sum_{j} \operatorname{deg}\left(f_{j}\right)+m-1\right) .
$$

Then $f_{1}, \ldots, f_{m}$ are linearly dependent.

Example 3.6. Infinitary versions of Corollary 3.5 fail.

(1) Since $\mathrm{St}_{2 n}$ is a polynomial identity for $M_{n}(\mathbb{F})$, the set

$$
S=\left\{\mathrm{St}_{2 n} \mid n \in \mathbb{N}\right\}
$$

is $M_{s}(\mathbb{F})$-locally linearly dependent (for every $s \in \mathbb{N}$ ), but is obviously not linearly dependent in $\mathbb{F}\langle\underline{X}\rangle$.

(2) To obtain an infinite set of polynomials in a bounded number of variables that is locally but not globally linearly dependent one just uses the set (3.3) together with the fact that the free algebra $\mathbb{F}\langle\underline{X}\rangle$ embeds into the free algebra $\mathbb{F}\langle X, Y\rangle$ on two variables [Coh, Section 2.5, Exercise 18] via

$$
\iota\left(X_{1}\right)=X, \quad \iota\left(X_{2}\right)=[X, Y], \ldots, \quad \iota\left(X_{n}\right)=\left[\iota\left(X_{n-1}\right), Y\right], \ldots
$$

3.2. Local directional linear dependence. We now turn to (local) directional linear dependence. The conclusion here is again that a finite set of locally directionally linearly dependent polynomials is indeed linearly dependent. However, the proof is somewhat more involved and the bounds obtained are worse.

Theorem 3.7. Let $\mathcal{A}$ be an algebra of linear operators. If $f_{1}, \ldots, f_{m} \in \mathbb{F}\left\langle X_{1}, \ldots, X_{n}\right\rangle$ are $\mathcal{A}$-locally directionally linearly dependent and $\mathcal{A}$ does not satisfy a polynomial identity of degree

$$
\gamma:=\frac{(d+1)(d+2)}{2}\left(m-1+\sum_{j} \operatorname{deg}\left(f_{j}\right)\right)+d, \quad \text { where } d=(m-1) m !,
$$

then $f_{1}, \ldots, f_{m}$ are linearly dependent.

Proof. Let $V$ be the space on which operators from $\mathcal{A}$ act. Choose $A_{i}, B_{j} \in \mathcal{A}$, $1 \leq i \leq n, i \leq j \leq m-1$. Let us consider

$$
H:=C_{2 m-1}\left(f_{1}(\underline{A}), \ldots, f_{m}(\underline{A}), B_{1}, \ldots, B_{m-1}\right) \in \mathcal{A} .
$$

By assumption, for each $v \in V$ the vectors $f_{1}(\underline{A}) v, \ldots, f_{m}(\underline{A}) v$ are linearly dependent. Hence by Lemma 2.3, the rank of $H$ is at most $(m-1) m !=: d$. So $H, H^{2}, \ldots, H^{d+1}$ are linearly dependent (cf. Remark 2.4 ). In particular,

$$
C_{2 d+1}\left(H, H^{2}, \ldots, H^{d+1}, D_{1}, \ldots, D_{d}\right)=0
$$

for all $D_{j} \in M_{s}(\mathbb{F})$. This shows that the polynomial

$$
g=C_{2 d+1}\left(h, h^{2}, \ldots, h^{d+1}, Z_{1}, \ldots, Z_{d}\right),
$$

where

$$
h=C_{2 m-1}\left(f_{1}, \ldots, f_{m}, Y_{1}, \ldots, Y_{m-1}\right)
$$

and $Y_{j}, Z_{j}$ are noncommuting indeterminates, is an identity of $\mathcal{A}$. Its degree is

$$
\frac{(d+1)(d+2)}{2} \operatorname{deg}(h)+d=\frac{(d+1)(d+2)}{2}\left(m-1+\sum_{j} \operatorname{deg}\left(f_{j}\right)\right)+d .
$$

According to our assumption this implies $g=0$. Repeating the argument based on Theorem 2.1 from the proof of Theorem 3.1 we see that $h, h^{2}, \ldots, h^{d+1}$ are linearly 
dependent polynomials. By comparing degrees in the $Y_{j}$, this is only possible if $h=$ 0. Applying Theorem 2.1 again, we obtain that $f_{1}, \ldots, f_{m}$ are linearly dependent.

We explicitly state the matrix version of Theorem 3.7:

Corollary 3.8. Let $f_{1}, \ldots, f_{m} \in \mathbb{F}\left\langle X_{1}, \ldots, X_{n}\right\rangle$ be $M_{s}(\mathbb{F})$-locally linearly dependent for some

$$
s>\frac{(d+1)(d+2)}{4}\left(m-1+\sum_{j} \operatorname{deg}\left(f_{j}\right)\right)+\frac{d}{2}, \quad \text { where } d=(m-1) m !,
$$

Then $f_{1}, \ldots, f_{m}$ are linearly dependent.

3.3. The Fock alternative. A representation theoretic proof (with some functional analytic flavor) of the local-global principles (over matrix algebras) can be given using the noncommutative Fock space. Note that the bounds obtained in this way are different from those given above in that they do not depend on the number of polynomials under consideration, but do depend on the number of variables appearing in our polynomials.

Proposition 3.9. Suppose $f_{1}, \ldots, f_{m} \in \mathbb{F}\left\langle X_{1}, \ldots, X_{n}\right\rangle$ be $M_{s}(\mathbb{F})$-locally directionally linearly dependent for

$$
s \geq \operatorname{dim} \mathbb{R}\left\langle X_{1}, \ldots, X_{n}\right\rangle_{k}=\sum_{i=0}^{k} n^{i}=: \sigma,
$$

where $k:=\max \left\{\operatorname{deg}\left(f_{j}\right) \mid j=1, \ldots, m\right\}$. Then $f_{1}, \ldots, f_{m}$ are linearly dependent.

Proof. The proof is based on the noncommutative Fock space. Define linear operators $S_{j}$ on $\mathbb{R}\left\langle X_{1}, \ldots, X_{n}\right\rangle_{k}$ by declaring, for a word $v \in\left\langle X_{1}, \ldots, X_{n}\right\rangle_{k}$ :

$$
S_{j} v= \begin{cases}X_{j} v & \operatorname{deg}(v)<k \\ 0 & \text { otherwise. }\end{cases}
$$

By construction, if $p \in \mathbb{R}\langle\underline{X}\rangle_{k}$, then

$$
p(\underline{S}) 1=p .
$$

(Note: The evaluation $\mathrm{ev}_{\underline{S}}$ yields a homomorphism $\mathbb{R}\left\langle X_{1}, \ldots, X_{n}\right\rangle \rightarrow M_{\sigma}(\mathbb{F})$ which is one-to-one when restricted to $\mathbb{R}\left\langle X_{1}, \ldots, X_{n}\right\rangle_{k}$.)

Now if $f_{1}, \ldots, f_{m}$ are $M_{s}(\mathbb{F})$-locally (directionally) linearly dependent, then by considering the directional evaluation at $(\underline{S}, 1)$, there exist scalars $\alpha_{m}$ not all zero satisfying

$$
0=\sum_{j=1}^{m} \alpha_{m} f_{m}(\underline{S}) 1=\sum_{j=1}^{m} \alpha_{m} f_{m}
$$

Hence the $f_{j}$ are linearly dependent.

3.4. Free algebras with involution. Often one is interested in evaluating polynomials at tuples of symmetric matrices, or is considering polynomials in disjoint tuples of variables $\underline{X}, \underline{X}^{*}$ with the obvious notion of evaluation. In these cases one considers one of the two notions of free algebras with involution (symmetric variables or free variables). All of the results given above have corresponding adaptations to free algebras with involution. The easy verifications are left as an exercise for the reader; the only nontrivial modifications are the results needed in the proofs. For instance, by [Sli], a polynomial of degree $<2 d$ cannot vanish on all symmetric $d \times d$ matrices. 


\section{REFERENCES}

[Ami] S. A. Amitsur, Generalized polynomial identities and pivotal monomials, Trans. Amer. Math. Soc. 114 (1965) 210-226. 3

[Aup] B. Aupetit, A primer on spectral theory, Universitext, Springer, 1991. 3

[BMM] K.I. Beidar, W.S. Martindale 3rd, A.V. Mikhalev, Rings with generalized identities, Marcel Dekker, Inc., 1996. 3

[BCM] M. Brešar, M. A. Chebotar, W. S. Martindale, Functional identities, Birkhäuser, 2007. 3

[BK] M. Brešar, I. Klep, Tracial Nullstellensätze, accepted for publication in the Borcea memorial volume, Birkhäuser, 2011. 3

[BŠ] M. Brešar, P. Šemrl, On locally linearly dependent operators and derivations, Trans. Amer. Math. Soc. 351 (1999) 1257-1275. 3

[CHSY] J.F. Camino, J.W. Helton, R.E. Skelton, J. Ye, Matrix inequalities: a symbolic procedure to determine convexity automatically, Integral Equations Operator Theory $\mathbf{4 6}$ (2003) 399-454. 1

[Coh] P. M. Cohn, Free ideal rings and localization in general rings, Cambridge University Press, 2006. 6

[DHM] H. Dym, J. W. Helton, S. McCullough: The Hessian of a non-commutative polynomial has numerous negative eigenvalues, J. Anal. Math. 102 (2007) 29-76. 1

[HHLM] D. M. Hay, J. W. Helton, A. Lim, S. McCullough: Non-commutative partial matrix convexity, Indiana Univ. Math. J. 57 (2008) 2815-2842. 1

[GHV] J. M. Greene, J.W. Helton, V. Vinnikov, Noncommutative plurisubharmonic polynomials Part I: global assumptions, preprint http://arxiv.org/abs/1101.0107 1

[HKM] J. W. Helton, I. Klep, S. McCullough, Tutorial on noncommutative convex algebraic geometry, to appear in: Semidefinite optimization and convex algebraic geometry, edited by B. Sturmfels et al. 1

[MŠ] R. Meshulam, P. Šemrl, Locally linearly dependent operators, Pacific J. Math. 203 (2002) 441-459. 3

[Pro] C. Procesi, Rings with polynomial identities, Marcel Dekker, Inc., 1973. 3

[Row] L. H. Rowen, Polynomial identities in ring theory, Academic Press, 1980. 3

[Sli] A. M. Sli'nko, Special varieties of Jordan algebras, Mat. Zametki 26 (1979) 337-344. 7

Faculty of Mathematics and Physics, University of Ljubljana, and, Faculty of Natural Sciences and Mathematics, University of Maribor, Slovenia

E-mail address: matej.bresar@fmf.uni-lj.si

E-mail address: igor.klep@fmf.uni-lj.si 